\title{
Revidiertes Mehrwertsteuergesetz: Wo liegen die Probleme?
}

Gabriela Lang

Korrespondenz:

Rechtsdienst FMH

Elfenstrasse 18

CH-3000 Bern 15

lex@fmh.ch

\section{Einleitung}

Das revidierte Mehrwertsteuergesetz (MWSTG) und die dazugehörige Ausführungsverordnung, beide seit dem 1. Januar 2010 in Kraft [1], wirken sich auch auf das Gesundheitswesen aus. In der im November 2010 erschienenen MWST-Branchen-Info 21 erläutert die Eidgenössische Steuerverwaltung die Gesetzgebung und gibt eine detaillierte Übersicht über die mehrwertsteuerpflichtigen oder von der Mehrwertsteuer befreiten Leistungen und Abgaben. Der folgende Artikel weist auf die wichtigsten Änderungen in der Broschüre hin und zeigt auf, wo aus Sicht der FMH Fallstricke liegen und welche Anliegen das MWSTG nicht erfüllt.

\section{Call-Center: mehrwertsteuerpflichtig}

Neu sind auch die von Ärztinnen und Ärzten erbrachten telemedizinischen Heilbehandlungen ausdrücklich von der Mehrwertsteuer ausgenommen. Allerdings fallen die durch die medizinischen Call-Center erbrachten Leistungen weiterhin unter die Steuerpflicht [2]. Dies erscheint uns nicht nur inkonsequent, sondern auch nicht mit der Förderung von eHealth vereinbar.

\section{Praxisgemeinschaften: Fallstricke}

Die Steuerbefreiung von als einfache Gesellschaft geführten Arztgemeinschaften untersteht nach wie vor strengen Bedingungen [3]. Neu und positiv: Es braucht keinen schriftlichen Gesellschaftsvertrag [4] mehr.

Alt und negativ bleibt der Fallstrick, dass wirklich alle Bedingungen zwingend erfüllt sein müssen, damit nicht der gesamte Umsatz auf einmal mehrwertsteuerpflichtig wird. Das stellt ein Klumpenrisiko dar, welches tendenziell für die Gruppenpraxis in Form einer Kapitalgesellschaft mit angestellten Ärzten spricht - auch wenn bei einer solchen wohl schnell die MWST-pflichtige Jahres-Umsatzgrenze von CHF 100000.- erreicht wird. Hingegen besteht das Risiko nicht mehr weiter, von bis zu 5 Jahren zurückliegenden Rückforderungen überrascht zu werden. So oder so greift hier aber die Mehrwertsteuer in die Freiheit der beteiligten Ärzte ein, ihre Praxisorganisation und deren Rechtsform selbst zu wählen [5].

Weiterhin mehrwertsteuerpflichtig ist die Lieferung von Medikamenten und medizinischen Hilfsmitteln der Gemeinschaft an die einzelnen Gesellschafter (Ärzte) oder an Dritte. Damit ist es der Gruppenpraxis faktisch verwehrt, den Medikamenteneinkauf gemeinsam zu tätigen, was aus Sicht der FMH unverständlich ist. Einmal mehr greift hier die Mehrwertsteuer in die ärztliche Praxisführung ein, wofür wir keinen sachlichen Grund erkennen können. Im gleichen Zug leuchtet nicht ein, dass beispielsweise Labor- oder Röntgenuntersuchungen für dritte Ärzte zur Mehrwertsteuerpflicht führen. Auch hier sollte es für die Befreiung genügen, dass die Tätigkeit selbst zur Untersuchung oder Behandlung von Patienten gehört.

\section{Ambulante Behandlungszentren}

Die Kriterien, wann ein ambulantes Behandlungszentrum mehrwertsteuermässig als Erbringer von Heilbehandlungen gilt, wurden gelockert - nebst der kantonalen Institutsbewilligung (sofern nach kantonalem Recht überhaupt erforderlich) muss gemäss Ziffer 5.1 der Brancheninfo neu gewährleistet sein, dass eine oder mehrere als Erbringer von Heilbehandlungen [...] anerkannte Personen zu 100\% anwesend sind [6]. Unseres Erachtens macht letzteres keinen Sinn: Denn was das Krankenversicherungsgesetz (Art. 36a KVG [7]), die Praxis des Bundesgerichts [8] und der Kanton als ambulantes Behandlungszentrum bzw. Institut anerkennen, soll auch von der Mehrwertsteuer akzeptiert werden. Es ist nicht nachvollziehbar, warum die Mehrwertsteuer hier zusätzliche Kriterien aufstellt.

\section{Gleichzeitige Tätigkeit in Gruppenpraxis und als Belegarzt}

Nach wie vor unbefriedigend gelöst ist die folgende Situation: Ein in einer Gruppenpraxis (in Form einer juristischen Person) angestellter Arzt arbeitet auch als Belegarzt in einem Spital, das dem Patienten die Gesamtrechnung stellt: Das Spital muss der Gruppenpraxis MWST für Personalausleihe bezahlen, was die Belegarzttätigkeit für das Spital nicht attraktiv macht.

\section{Hinweis für Gruppenpraxen}

Aufgrund der vielen Besonderheiten im revidierten Mehrwertsteuergesetz empfehlen wir Ihnen, Ihre Situation von Fachleuten sorgfältig prüfen zu lassen, beispielsweise mit Beratern der FMH Consulting Services. Die FMH stellt ihren Mitgliedern auf myFMH Musterverträge für einfache Gesellschaften und für die Nutzung von Infrastrukturen zur Verfügung, welche von Fall zu Fall an die persönlichen Gegebenheiten angepasst werden können. 
Unseres Erachtens ist es nicht richtig, dass die Eidgenössische Steuerverwaltung hier aufgrund des Kriteriums der Personalausleihe eine MWST-Pflicht begründet. Richtigerweise soll auch die Tätigkeit am Belegspital von der MWST befreit sein, weil der Belegarzt ärztliche Untersuchung und Behandlungen erbringt, die ja gemäss Gesetz von der MWST ausgenommen sein sollen [9].

\section{Umsatzgrenze für das Saldosteuerverfahren zu tief}

Ein weiteres Problem ist die Begrenzung des MWSTpflichtigen Umsatzes auf CHF 5000000 .- beim Saldosteuerverfahren. Darüber hinaus muss das effektive Verfahren oder das Pauschalsteuerverfahren gewählt werden, was sehr viel mehr Aufwand gibt. Ein Umsatz von 5 Millionen ist schnell erreicht - 10 Grundversorger in einer Gruppenpraxis mit direkter Medikamentenabgabe überschreiten die Grenze. Der MWSTpflichtige Umsatz für das Saldosteuerverfahren sollte nach oben offen sein.

\section{Nicht erfüllte Anliegen der FMH}

Die neue MWST-Gesetzgebung nimmt die ärztliche Untersuchung und Behandlung von der Mehrwertsteuer aus. Dieser Grundsatz muss aus Sicht der FMH konsequent respektiert werden. Die oben erwähnten Beispiele zeigen, dass noch Korrekturbedarf besteht: Entscheidend soll allein das Kriterium sein, ob eine ärztliche Untersuchung oder Behandlung eines Patienten gegeben ist. Das Setting darf keine Rolle spielen. Gruppenpraxen (Ehepaare, einfache Gesellschaften, GmbH, AG usw.) und Belegarzttätigkeit sind bezüglich MWST gleich zu behandeln wie Einzelpraxen. Praxisformen, die gemäss KVG, kantonalem Gesundheitsrecht und Gerichtspraxis möglich sind, darf die MWST nicht faktisch verhindern. Die Mehrwertsteuer soll nicht Gesundheitspolitik betreiben und schon gar nicht die Ziele der aktuellen Gesundheitspolitik unterlaufen.

\section{Anmerkungen}

1 Einen Überblick über die wichtigsten Änderungen und die damit verbundenen Auswirkungen auf die Mehrwertsteuerpflicht von Arztpraxen haben Lucia Schlemmer und Hanspeter Kuhn Ende 2009 in der Schweizerischen Ärztezeitung bereits gegeben (SÄZ 2009;90: 51/52).

2 Die ESTV stützt ihre Begründung auf ein Urteil des Bundesverwaltungsgerichts vom 24. Februar 2009 (A-3395/2007), wonach eine von der Steuer ausgenommene Heilbehandlung im Sinne von Art. 18 Ziff. 3 aMWSTG (Art. 21 Abs. 2 Ziff. 3 nMWSTG) eine besondere physische Nähe, d.h. die Behandlung direkt am Patienten voraussetzt. Dies sei bei einer telefonischen Beratung offensichtlich nicht der Fall. Diese Logik überzeugt uns nicht, denn auch die telemedizinische Konsultation durch Arzt oder ärztliches Call-Center ist Heilbehandlung (gegenüber beiden kann ein allfälliger Behandlungsfehler eingeklagt werden).

3 Es gelten unter anderem folgende Bedingungen (siehe auch Gérard Dustour, MWSt-Risiko und Gruppenpraxen, in: SÄZ 2008;89:46):

1. Die Gemeinschaft wird ausschliesslich zum Zwecke der Beschaffung der erforderlichen Infrastruktur (z.B. medizinischer Geräte, Praxisräumlichkeiten, Personal) sowie des damit verbundenen Betriebes gebildet.

2. Alle Gesellschafter (Ärztinnen und Ärzte) der einfachen Gesellschaft sind natürliche Personen (Personengesellschaften und juristische Personen sind ausgeschlossen) und müssen einen Beruf im Gesundheitswesen ausüben sowie über die kantonale Bewilligung zur selbständigen Berufsausübung verfügen.

3. Die anfallenden Kosten werden den einzelnen Gesellschaftern von der Gemeinschaft anteilsmässig zu Selbstkosten (ohne Gewinnzuschlag) in Rechnung gestellt.

4. Dritten gegenüber werden durch die Gemeinschaft weder Dienstleistungen erbracht noch Gegenstände geliefert.

4 V.a. bei Ehepaar-Gruppenpraxen war dieses Kriterium für die Befreiung von der Mehrwertsteuer ausgesprochen künstlich und verursachte praktische Probleme.

5 vgl. BGE 9C_701/2008 vom 20. April 2009, Erw. 4.4.

6 Unter altem Recht wurde hierzu in der Branchenbroschüre Nr. 20 noch verlangt, dass

- deren Leiter oder Leiterin als Leistungserbringer/ in von Heilbehandlungen gilt (die Leitung muss entweder fest angestellt sein oder es muss vertraglich eine 100\%ige Anwesenheit einer solchen Leitung gewährleistet sein) oder

- bei denen alle im Zentrum Behandlungen ausführenden Teilhaber einer juristischen Person oder Personengesellschaft (z.B. Gesellschafter, Aktionäre) als Leistungserbringer von Heilbehandlungen gelten (dies gilt nur, sofern keine gegen aussen bekanntgegebene Leitung vorliegt).

7 Art. 36a KVG lautet: «Einrichtungen, die der ambulanten Krankenpflege durch Ärzte und Ärztinnen dienen, sind zugelassen, wenn die dort tätigen Ärzte und Ärztinnen die Voraussetzungen nach Artikel 36 erfüllen.»

8 Gemäss Bundesgerichtsentscheid 9C_701/2008 vom 20. April 2009 gilt Art. 36a KVG für juristische Personen, welche Ärzte anstellen (ohne dem HMO-Modell zu folgen) als auch für Praxisorganisationen, wonach die juristische Person lediglich die Infrastruktur oder andere Dienstleistungen für mehrere Ärzte zur Verfügung stellt, diese aber als Einzelunternehmer tätig sind und nur die Dienstleistungen von der Gesellschaft beziehen (vgl. Erw. 4.3 und 4.4).

9 Im Unterschied beispielsweise zur outgesourcten IT bei der leuchtet ein, dass die IT-Unternehmung dem Spital MWST verlangen muss, erbringt doch das IT-Personal keine Patientenbehandlung. 